A. Herbert $\cdot$ C. Liu $\cdot$ S. Karamohamed . J. Schiller · J. Liu $\cdot$ Q. Yang $\cdot$ P. W. F. Wilson · L. A. Cupples $\cdot$ J. B. Meigs

\title{
The -174 IL-6 GG genotype is associated with a reduced risk of type 2 diabetes mellitus in a family sample from the National Heart, Lung and Blood Institute's Framingham Heart Study
}

Received: 19 May 2004 / Accepted: 18 April 2005 / Published online: 17 June 2005

(C) Springer-Verlag 2005

\begin{abstract}
Aims/hypothesis: We evaluated the -174 IL-6 gene polymorphism as a risk factor for type 2 diabetes mellitus in a family-based analysis. Methods: We tested for associations between the $-174 \mathrm{IL}-6 \mathrm{G} / \mathrm{C}$ promoter polymorphism and fasting plasma glucose (FPG) and type 2 diabetes in a sample of 1,428 individuals from the largest 182 families in the National Heart, Lung and Blood Institute's Framingham Heart Study population. Results: A significant association was found with FPG $(p=0.01)$ and $\log (\mathrm{FPG})(p=0.005)$ using a modified family-based transmission disequilibrium test, the family-based association test (FBAT). The association between IL-6 genotype and FPG $(p=0.035)$ and $\log (\mathrm{FPG})(p=0.03)$ was also found in the subset of families that were informative in FBAT using
\end{abstract}

\footnotetext{
A. Herbert

Department of Genetics and Genomics,

Boston University School of Medicine,

Boston, MA, USA
}

A. Herbert $(\bowtie) \cdot$ C. Liu · S. Karamohamed ·

J. Schiller · J. Liu

Framingham Heart Study Genetics Laboratory,

Department of Neurology,

Boston University School of Medicine,

715 Albany Street,

Boston, MA, 02118, USA

e-mail: aherbert@bu.edu

Q. Yang · L. A. Cupples

Department of Biostatistics,

Boston University School of Public Health,

Boston, MA, USA

P. W. F. Wilson

Department of Medicine,

Boston University School of Medicine,

Boston, MA, USA

\section{J. B. Meigs}

General Internal Medicine and Clinical

Epidemiology Units, General Medicine Division,

Department of Medicine,

Massachusetts General Hospital and Harvard Medical School,

Boston, MA, USA a mixed-effects regression model and strengthened after adjustment for potential confounders ( $p=0.008$ for $\log$ [FPG]). The mean glucose level estimated from models with log (FPG) as the dependent variable for the GG genotype in the informative families was significantly lower $(5.20 \pm 0.06$ $\mathrm{mmol} / \mathrm{l})$ than for the GC $(5.41 \pm 0.06 \mathrm{mmol} / \mathrm{l})$ and $\mathrm{CC}(5.38 \pm$ $0.06 \mathrm{mmol} / \mathrm{l}$ ) genotypes ( $p=0.03$ for contrast between $\mathrm{GG}$ and GC genotypes). In the subset of informative families, the risk of type 2 diabetes associated with the GG genotype was lower relative to the $\mathrm{GC}$ and $\mathrm{CC}$ genotypes combined (potential confounder-adjusted, mixed-effects odds ratio $0.35,95 \%$ CI $0.14-0.88, p=0.026$, unaffected $n=391$, affected $n=32$ ). Conclusions/interpretation: These results are consistent with a protective role for the -174 IL-6 G allele against type 2 diabetes and warrant further analysis of this polymorphism.

Keywords Association · Family IL-6 · -174 polymorphism - Type 2 diabetes

Abbreviations FBAT: family-based association test . FHS: Framingham Heart Study - FPG: fasting plasma glucose $\cdot$ OR: odds ratio

\section{Introduction}

The -174 IL- 6 gene polymorphism has been proposed as a risk factor for type 2 diabetes mellitus based on studies of unrelated individuals. These results have been conflicting. A study of 33 Caucasian subjects found that CC homozygotes had a lower post-oral-challenge glucose level, a lower $\mathrm{HbA}_{1} \mathrm{c}$ value, and a lower fasting insulin level than either CG or GG genotypes [1]. In native American and Caucasian populations, the GG genotype has also been associated with increased risk of type 2 diabetes [2], but in a Finnish population it has been associated with increased insulin sensitivity (and presumably lower type 2 diabetes risk) [3]. In another study of unrelated obese individuals, the CC genotype has been reported to carry an increased risk of type 2 diabetes [4]. 
Table 1 Descriptive statistics with -174 IL-6 genotypes and fasting plasma glucose (FPG) for Framingham Heart Study subjects in the family sample

Unless otherwise stated, values are mean $\pm \mathrm{SD}$ $F P G$ fasting plasma glucose

\begin{tabular}{llll}
\hline & Men $(n=328)$ & Women $(n=342)$ & $p$-value of contrast \\
\hline Age (years) & $50.50 \pm 9.97$ & $52.49 \pm 10.29$ & 0.01 \\
Alcohol use (g/week) & $137.21 \pm 111.99$ & $76.54 \pm 48.76$ & $<0.0001$ \\
Cigarettes (number/day) & $4.17 \pm 10.10$ & $3.33 \pm 8.44$ & 0.25 \\
Physical activity index & $37.44 \pm 7.61$ & $37.25 \pm 5.88$ & 0.72 \\
BMI (kg/m $\left.{ }^{2}\right)$ & $28.27 \pm 4.30$ & $26.99 \pm 6.13$ & 0.002 \\
FPG $(\mathrm{mmol} / \mathrm{l})$ & $5.70 \pm 1.65$ & $5.43 \pm 1.63$ & 0.04 \\
CC $(\%)$ & 13.72 & 15.20 & 0.59 \\
CG $(\%)$ & 48.48 & 44.74 & 0.33 \\
GG $(\%)$ & 37.80 & 40.06 & 0.55 \\
\hline
\end{tabular}

Here, we tested for associations between the -174 IL-6 $\mathrm{G} / \mathrm{C}$ promoter polymorphism and fasting plasma glucose (FPG) and type 2 diabetes in a family-based sample from the National Heart, Lung and Blood Institute's Framingham Heart Study (FHS) where over 14\% of participants meet clinical criteria for the diagnosis of diabetes by age 70 [5].

\section{Subjects, materials and methods}

Subjects The FHS began in 1948 with a randomly selected cohort from the town of Framingham, MA. Initially 5,209 participants were enrolled. In 1971, 5,124 offspring and their spouses were added [6]. From this population-based sample, we selected the largest 182 pedigrees for study. This study was approved by the Boston University IRB and written informed consent was obtained for each subject.

Genotyping Participants were genotyped for the -174 polymorphism using a mass spectroscopy-based singlenucleotide polymorphism detection assay (Sequenom, San Diego, CA, USA) [7]. PCR primers were 5'ACGTTG GATGAGCCTCAATGACGACCTAAG and 5'ACGTTG GATGGATTGTGCAATGTGACGTCC and the extension primer was 5'CCCCCTAGTTGTGTCTTGC.

Traits FPG was measured with a hexokinase reagent kit (A-Gent Glucose Test; Abbott, South Pasadena, CA, USA) at Framingham Offspring Study examination cycle 5 (1991$1995)$ on 1,370 Offspring participants. Glucose assays were run in duplicate; the intra-assay $\mathrm{CV}$ was $<3 \%$. Type 2 diabetes status was defined as diabetes treatment, an FPG level $\geq 7.0 \mathrm{mmol} / \mathrm{l}$ on at least two of five Offspring examinations, or a $2-\mathrm{h}$ post-challenge glucose level $\geq 11.1 \mathrm{mmol} / 1$ at examination 5, excluding known type 1 diabetes [8].

Statistical analysis The 182 extended pedigrees consisted of 826 nuclear families with 2,810 persons of whom 670 had been both genotyped and phenotyped. We used a generalised transmission disequilibrium test, implemented in the family-based association test (FBAT) program, or mixed-effects regression models (that account for relatedness among observations) to test for association between the -174 IL-6 G/C promoter polymorphism and FPG [9]. Log-transformed FPG was also analysed as the dataset was right-skewed. In brief, FBAT excludes from analysis those families where both parents are homozygotes because all offspring have the same genotype. In FBAT, a score for each family is calculated by multiplying an adjusted offspring trait value (mean $=0$ ) by a genotypic value (e.g. 1 if a genotype is present, 0 otherwise). The program determines the expected scores under the null hypothesis of no association or no linkage by conditioning on offspring trait values and parental genotypes, assuming Mendelian transmission of alleles to children [9]. The difference between the observed and expected score is summed over all the families (this equals 0 for families where both parents are homozygotes and offspring share the same genotype), and corrects for genotypic correlations among siblings by using a White empirical variance-covariance estimator when there is evidence for linkage [10]. These values are used to calculate a test statistic which has a chi square distribution [9]. The direction of an effect can be determined by the sign of the test statistic, with negative scores arising when a genotype is associated with trait values less than the population-adjusted mean and positive values when it is higher. FBAT was performed using measured values of FPG without adjustment, using the offset option to centre the trait distribution and increase the power of the analysis. Results from the FBAT analysis were corroborated in the family sample with a mixedeffects model in PROC MIXED (SAS Institute) to estimate genotypic means, using empirical covariance matrix structures to account for correlation within the data. This strategy examines association, but does not evaluate transmission of alleles as does FBAT. This approach also allows for covariate adjustment to improve estimates of effect size.

Table 2 Global $p$ values for association of -174 IL-6 genotypes with type 2 diabetes mellitus (DM2) and the log of unadjusted fasting plasma glucose (FPG) determined using the family-based association test (FBAT) under an additive model and PROC MIXED where each genotype was modelled separately

\begin{tabular}{lllccl}
\hline & $\begin{array}{l}\text { FBAT } \\
p \text {-value }\end{array}$ & $z$-score & & $\begin{array}{l}\text { Informative } \\
\text { families }(n)\end{array}$ & $\begin{array}{l}\text { PROC-MIXED } \\
p \text {-value }\end{array}$ \\
\hline DM2 & 0.34 & -0.96 & 0.96 & 96 & - \\
FPG & 0.005 & -2.78 & 2.78 & 143 & 0.03 \\
\hline
\end{tabular}

The $z$-score for each allele and the number of informative nuclear families contributing to the result are also displayed. The major allele is $\mathrm{G}$, the minor allele $\mathrm{C}$ 
Table 3 Descriptive statistics for Framingham Heart Study subjects from the informative families used in this study and analysed by the family-based association test

Unless otherwise stated, values are mean $\pm \mathrm{SD}$ $F P G$ fasting plasma glucose

\begin{tabular}{llll}
\hline & Men $(n=218)$ & Women $(n=232)$ & $p$ value of contrast \\
\hline Age (years) & $50.94 \pm 10.20$ & $52.44 \pm 10.50$ & 0.11 \\
Alcohol use (g/week) & $134.94 \pm 113.97$ & $64.35 \pm 42.24$ & $<0.0001$ \\
Cigarettes (number/day) & $4.13 \pm 10.26$ & $3.52 \pm 8.74$ & 0.48 \\
Physical activity index & $37.47 \pm 7.88$ & $37.10 \pm 6.08$ & 0.56 \\
BMI $\left(\mathrm{kg} / \mathrm{m}^{2}\right)$ & $28.01 \pm 4.48$ & $26.73 \pm 5.68$ & 0.005 \\
FPG $(\mathrm{mmol} / \mathrm{l})$ & $5.52 \pm 1.09$ & $5.33 \pm 1.47$ & 0.05 \\
CC $(\%)$ & 17.17 & 16.10 & 0.92 \\
CG $(\%)$ & 50.62 & 49.48 & 0.52 \\
GG $(\%)$ & 32.18 & 34.56 & 0.24 \\
\hline
\end{tabular}

We report results from a model without any covariate adjustment and also from a model that adjusts for the potential confounding variables of age (including squared and cubed terms to allow for non-linearity), sex, smoking, physical activity, alcohol use and oestrogen use in women, derived from separate regression models for each sex as previously described [5]. Odds ratios (ORs) for the discrete diabetes phenotype were calculated in a logistic regression model for both the whole sample and the informative family set using a recessive model. We used PROC GENMOD, allowing for correlated observations among family members and using these same covariates as described for PROC MIXED models.

\section{Results and discussion}

Characteristics of subjects from all study families are shown in Table 1. Using FBAT, a substantial association with FPG $(p=0.01)$ and $\log (\mathrm{FPG})(p=0.005)$ measured at Framingham Offspring Study examination 5 (1991-1995) was found for the IL-6 polymorphism (Table 2). The negative $z$-score for the $\mathrm{G}$ allele in both analyses (FBAT Score Statistic -2.6 and -2.8 ) indicates that it is associated with lower FPG levels relative to the population mean. Adjustment for BMI did not significantly alter this result, indicating that the IL-6 genotype was not acting as a surrogate marker of obesity (Table 2). We also tested for an association between the IL-6 polymorphism and type 2 diabetes affection status. In the whole family sample, $10.3 \%$ of men $(n=34)$ and $8.7 \%$ of women $(n=30)$ were classified as diabetic. We did not find an association of
-174 IL-6 genotype with type 2 diabetes when all study families were examined using FBAT (Table 2). The $z$-score for the GG genotype tended to be negative, suggesting that the GG genotype may be potentially protective against type 2 diabetes and consistent with our results on FPG.

We also examined the family-based data in a mixedeffects model implemented in PROC MIXED to validate the association between IL-6 genotype and FPG using a different statistical methodology. We further examined the association with log-transformed FPG to test for an underlying lognormal distribution of data, as is commonly true for complex biological interactions. In the entire family dataset, we found no significant association of IL-6 genotype with FPG or log (FPG) levels. One possibility for the difference in results between FBAT and the mixed-effects regression model in the family set is that FBAT uses only a limited set of families for analysis, rather than the whole set. In FBAT, families where both parents are homozygous are discarded from the analysis. In these families, the trait variability among offspring cannot be due to variation at the IL-6 locus as all children share the same genotype. Thus, only families that yield information about the effect of the IL-6 polymorphism on trait values are analysed. In contrast, the analysis using PROC MIXED included all families, even those where variability was not due to the IL-6 locus. When we limited the analysis in PROC MIXED to the 144 families that were informative in FBAT (Table 3), we found a significant association between the -174 IL-6 polymorphism FPG $(p=0.035)$ (Table 2$)$ and $\log (\mathrm{FPG})(p=0.03)$. When potentially confounding variables were included in the model, the association strengthened $(p=0.008)$. Sex-specific analysis in

along with the mean value and SE for each genotype of fasting plasma glucose (FPG) (in $\mathrm{mmol} / \mathrm{l}$ )

Table 4 The number of individuals with each genotype in the whole family set and the subset that was informative in the familybased transmission disequilibrium test from the Offspring Cohort,

\begin{tabular}{|c|c|c|c|c|c|c|c|c|}
\hline & \multicolumn{2}{|c|}{ Number } & \multicolumn{2}{|c|}{ Mean FPG } & \multicolumn{2}{|l|}{$\mathrm{SE}$} & \multicolumn{2}{|c|}{$p$ value } \\
\hline & All & Informative & All & Informative & All & Informative & All & Informative \\
\hline All & 670 & 450 & 5.42 & 5.33 & 0.06 & 0.06 & 0.49 & 0.032 \\
\hline GG & 261 & 150 & 5.39 & 5.20 & 0.06 & 0.06 & - & - \\
\hline GC & 312 & 225 & 5.47 & 5.41 & 0.06 & 0.06 & 0.71 & 0.026 \\
\hline $\mathrm{CC}$ & 97 & 75 & 5.32 & 5.38 & 0.06 & 0.06 & 0.87 & 0.39 \\
\hline
\end{tabular}

FPG was estimated from models using $\log (\mathrm{FPG})$ as the dependent variable and without covariate adjustment. The major allele is $\mathrm{G}$, the minor allele C. Global $p$ values are in the column labelled All. The other $p$ values are contrasts between the GG and other genotypes 
these models indicated that the association with -174 IL-6 genotype was stronger in females $(p=0.01$, contrast between GG and GC genotype mean levels, $p=0.01$ ) than in males $(p=0.15$, contrast between GG and GC genotype mean levels, $p=0.06$ ).

Comparison of the mean FPG levels in the sample of informative families showed that the GG genotype was associated with lower values than either the GC or CC genotypes (Table 4), indicating a recessive model of transmission. These findings are consistent with those of a Finnish study, which found an association between the GG genotype and increased insulin sensitivity, suggesting that the GG genotype may protect against the development of type 2 diabetes [3]. We calculated the risk of type 2 diabetes for the GG genotype relative to the $\mathrm{GC}$ and $\mathrm{CC}$ genotypes combined (i.e. treating the $\mathrm{G}$ allele as recessive) in the same sample of informative families (unaffected $n=391$, affected $n=32$ ). No significant association was found in an unadjusted analysis $(\mathrm{OR}=0.62$, CI $0.28-1.34$ $p=0.22$ ), mirroring the FBAT analysis. This result was not unexpected as discrete traits with two groupings are often less informative than continuous variables such as FPG. With the regression-based approach, it is possible to incorporate other predictors for type 2 diabetes into the model to improve analytical power. When covariates (described in the Materials and methods) were included in the model to account for the effect of potential confounding variables, the GG genotype was associated with a protective effect on risk of diabetes (adjusted $\mathrm{OR}=0.35,95 \%$ CI $0.14-0.88$, $p=0.026$ ). These results are consistent with the increased risk of type 2 diabetes in individuals with the $\mathrm{CC}$ genotype observed in one study [4] but differ from findings in Pima Indians [2]. It is possible that the Pima study was potentially confounded by population admixture as fullheritage Native Americans have only the $\mathrm{G}$ allele at the -174 IL-6 locus [2]. It is also possible that the -174 IL-6 susceptibility to type 2 diabetes differs in Caucasians, reflecting the action of other genes. Given these conflicting results, and the relatively small number of diabetic subjects in our sample, additional studies are needed to confirm the impact of the -174 IL- 6 on type 2 diabetes.

Our results in this family sample reveal an association between the -174 IL- 6 promoter genotype and levels of FPG. They illustrate the advantage of using family-based tests where non-informative individuals can be identified and excluded from analysis. In certain situations such as we describe here, it may thus be possible to detect effects that might be missed in an unrelated sample where the noise due to action of other genes or unmeasured environmental effects obscure the signal from the gene of interest. Here, statistically significant associations using mixed-effects regression models were obtained only after reducing sample heterogeneity by excluding families in which both parents were homozygous at the IL-6 locus. These mixed-effects regression models were then useful for analysing the relationship between type 2 diabetes and the -174 IL- 6 polymorphism in the subset of informative families. Together these results reveal the complexities in determining the impact of genetic polymorphisms on quantitative traits in cohorts of unrelated individuals where sources of genetic heterogeneity cannot be controlled for in the same way as they can in families. Our results suggest that the GG -174 IL-6 genotype is associated with lower FPG levels and is protective against type 2 diabetes. Examining the molecular basis for this association will require additional research.

Acknowledgements This work was supported by the National Heart, Lung and Blood Institute's FHS (Contract No. N01-HC25195). Support was also received from NIH grant HL64753 with E. Benjamin as Principal Investigator. This study also received support in the form of a grant from the American Diabetes Association (A. Herbert, Principal Investigator). J. B. Meigs is supported in part by a Career Development Award from the American Diabetes Association. We thank the staff of the FHS Genetics Laboratory for work performed to support this study.

\section{References}

1. Fernandez-Real JM, Broch M, Vendrell J et al (2000) Interleukin-6 gene polymorphism and insulin sensitivity. Diabetes 49:517-520

2. Vozarova B, Fernandez-Real JM, Knowler WC et al (2003) The interleukin-6 (-174) G/C promoter polymorphism is associated with type- 2 diabetes mellitus in native Americans and Caucasians. Hum Genet 112:409-413

3. Kubaszek A, Pihlajamaki J, Punnonen K, Karhapaa P, Vauhkonen I, Laakso M (2003) The C-174G promoter polymorphism of the IL-6 gene affects energy expenditure and insulin sensitivity. Diabetes 52:558-561

4. Mohlig M, Boeing H, Spranger J et al (2004) Body mass index and $\mathrm{C}-174 \mathrm{G}$ interleukin-6 promoter polymorphism interact in predicting type 2 diabetes. J Clin Endocrinol Metab 89:18851890

5. Meigs JB, Panhuysen CI, Myers RH, Wilson PW, Cupples LA (2002) A genome-wide scan for loci linked to plasma levels of glucose and $\mathrm{HbA}(1 \mathrm{c})$ in a community-based sample of Caucasian pedigrees: the Framingham Offspring Study. Diabetes $51: 833-840$

6. Kannel WB, Feinleib M, McNamara PM, Garrison RJ, Castelli WP (1979) An investigation of coronary heart disease in families. The Framingham Offspring Study. Am J Epidemiol 110:281-290

7. Buetow KH, Edmonson M, MacDonald R et al (2001) Highthroughput development and characterization of a genomewide collection of gene-based single nucleotide polymorphism markers by chip-based matrix-assisted laser desorption/ionization time-of-flight mass spectrometry. Proc Natl Acad Sci U S A 98:581-584

8. Meigs JB, Cupples LA, Wilson PW (2000) Parental transmission of type 2 diabetes: the Framingham Offspring Study. Diabetes 49:2201-2207

9. Horvath S, Xu X, Laird NM (2001) The family based association test method: strategies for studying general genotype-phenotype associations. Eur J Hum Genet 9:301-306

10. White H (1980) A heteroskedasticity-consistent covariance matrix estimator and a direct test for heteroskedasticity. Econometrica 48:817-838 